\title{
VARIABILIDAD GENOTÍPICA EN MAÍZ NATIVO PARA EFICIENCIA Y RESPUESTA A FÓSFORO EN ETAPA DE FLORACIÓN
}

\section{NATIVE MAIZE GENOTYPIC VARIABILITY FOR PHOSPHORUS EFFICIENCY AND RESPONSIVENESS AT THE FLOWERING STAGE}

\author{
Enrique Flores-Torres ${ }^{1}$, Iván Ochoa-Cadavid ${ }^{2}$, Ricardo E. \\ Preciado-Ortíz ${ }^{3}$ y Jeannette S. Bayuelo-Jiménez ${ }^{1 *}$
}

\begin{abstract}
'Instituto de Investigaciones Agropecuarias y Forestales, Unidad San Juanito Itzícuaro, Universidad Michoacana de San Nicolás de Hidalgo. Km. 9.5 Carr. Morelia-Zinapécuaro. 58880, Tarímbaro, Michoacán, México. ²Subdirección de Investigación y Desarrollo, Unipalma S.A., Calle 74 A No. $22-31$. Bogotá, Colombia. ${ }^{3}$ Instituto Nacional de Investigaciones Forestales, Agrícolas y Pecuarias. Km 6.5 Carr. Celaya. 38110, San Miguel de Allende, Celaya, Guanajuato. México.
\end{abstract}

*Autor para correspondencia (bayuelo@umich.mx)

\section{RESUMEN}

La reducida disponibilidad de fósforo $(P)$ en el suelo limita el rendimiento de los cultivos. El desarrollo de cultivares con tolerancia a la deficiencia de $\mathrm{P}$ puede representar una solución más sostenible que la que depende solamente de la aplicación de fertilizantes fosforados. Se estudió la variabilidad genotípica de maíz (Zea mays L.) de la Meseta P'urhépecha en Michoacán, México para eficiencia y respuesta a P, en la etapa de floración. El estudio incluyó cuatro localidades con 27 genotipos cultivados en suelos con deficiencia de $\mathrm{P}$, con una dosis de fertilización reducida $\left(25 \mathrm{~kg} \mathrm{P}_{2} \mathrm{O}_{5}\right.$ ha-1) (BP) y alta $\left(50 \mathrm{~kg} \mathrm{P}_{2} \mathrm{O}_{5} \mathrm{ha}^{-1}\right)$ (AP). Los genotipos de maíz difirieron en crecimiento vegetativo y eficiencia a $P$, definida como el crecimiento sostenido en condiciones de baja disponibilidad de P. Los 27 genotipos se agruparon en tres categorías de eficiencia con base en sus parámetros de crecimiento en BP y sus valores relativos al tratamiento de AP y en cuatro categorías de acuerdo con el valor del índice de eficiencia a $\mathrm{P}$ en combinación con la respuesta en crecimiento en AP. La distribución de los genotipos entre los tres grupos de eficiencia y respuesta a $P$ fue estable en todas las localidades. Los genotipos SHUI-2 (6), ZR-6 (75), Paso del Muerto (113), Tepetate (206) y Macho II-04 (230) fueron los más eficientes en BP y los que respondieron a la alta fertilización. Los genotipos eficientes tuvieron una mayor producción de materia seca y contenido de $\mathrm{P}$ en la planta y una mayor eficiencia en la absorción de P. La maduración tardía de los genotipos eficientes propició un incremento en la adquisición de P del suelo y mayor crecimiento en BP. Estos resultados indican que existe variabilidad genotípica para la eficiencia y respuesta a $\mathrm{P}$ en el germoplasma de maíz.

Palabras clave: Zea mays, crecimiento, eficiencia en absorción de P, madurez tardía, suelos ácidos.

\section{SUMMARY}

Phosphorus $(P)$ deficiency can severely limit maize yield. Efforts to develop cultivars with tolerance to $\mathrm{P}$ deficiency represent a solution which is more sustainable than sole reliance on $\mathrm{P}$ fertilizer application. This study evaluated genotypic variability in $\mathrm{P}$ efficiency and response at the flowering stage of maize (Zea mays L.) accessions from the P'urhépecha Plateau, at Michoacán, México. Twenty-seven accessions were grown on P-deficient andosol, at four locations with low $\left(25 \mathrm{~kg} \mathrm{P}_{2} \mathrm{O}_{5} \mathrm{ha}^{-1}\right)$ and high $\left(50 \mathrm{~kg} \mathrm{P}_{2} \mathrm{O}_{3}\right.$ ha $\left.^{-1}\right) \mathrm{P}$ fertilization under rain-fed conditions. The accessions differed greatly in their growth and $\mathrm{P}$ efficiency, defined as growth under suboptimal $\mathrm{P}$ availability. They were divided into three categories based on $P$ efficiency, and four categories according to $P$ efficiency in combination with high $P$ response. The distribution of accessions among three $\mathrm{P}$ efficiency classes was stable across locations.
Accessions SHUI-2 (6), ZR-6 (75), Paso del Muerto (113), Tepetate (206) and Macho II-04 (230) had the highest efficiency under low P and also responded to high $\mathrm{P}$ fertilization. P-efficient accessions had greater biomass, $\mathrm{P}$ tissue content and $\mathrm{P}$ acquisition efficiency (PAE) under $\mathrm{P}$ deficiency. Late maturity of $P$ efficient genotypes improved PAE and growth under low $P$. These results indicate that maize landraces from the P'urhépecha Plateau exhibit genotypic variation for $P$ efficiency and $P$ responsiveness.

Index words: Zea mays, plant growth, P acquisition efficiency, late maturity, acid soils

\section{INTRODUCCIÓN}

La reducida disponibilidad de fósforo $(P)$ en el suelo es uno de los principales factores que limitan el crecimiento y rendimiento de los cultivos mundialmente (Lynch, 2013). Alcalá et al. (2001) señalan que en andosoles, la reducida disponibilidad de $\mathrm{P}\left(<10 \mathrm{mg} \mathrm{kg}^{-1}\right)$ y la alta capacidad de retención de fosfatos (> $85 \%$ ), debido a la alta composición mineral de óxidos de Fe y Al, son factores limitantes en la producción de maíz (Zea mays L.) de la Meseta P'urhépecha. La sostenibilidad de los sistemas agrícolas de esta región, requiere de la identificación y desarrollo de genotipos eficientes para adquirir y utilizar el $\mathrm{P}$ sea del suelo o del fertilizante (Lynch, 2013).

El mejoramiento genético de la eficiencia en el uso de P (EUP), definida como el incremento de la producción de materia seca o rendimiento por unidad del nutriente absorbido (Wang et al., 2010) se ha realizado con variedades mejoradas derivadas de un reducido acervo de germoplasma y predominantemente adaptadas a una agricultura intensiva y ambientes óptimos (Manschadi et al., 2014). En contraste, las variedades tradicionales de maíz que se han desarrollado en ambientes específicos o marginados con reducida disponibilidad de nutrientes, representan una fuente importante de variación para la selección de genotipos adaptados a sistemas rurales (Newton et al., 2010). Por 
ejemplo, las variedades nativas de la Meseta P'urhépecha están adaptadas a los ambientes de reducida fertilidad y poseen características en la raíz que no son comunes en el germoplasma élite (Bayuelo-Jiménez et al., 2011). Las variedades eficientes a $P$ poseen raíces axilares (seminales 0 nodales) más superficiales y ramificadas que incrementan la absorción de P en la superficie del suelo y el crecimiento durante la etapa de plántula (Bayuelo-Jiménez et al., 2012) y etapa vegetativa (Bayuelo-Jiménez et al., 2011). A pesar de estos hallazgos, no existen estudios sistemáticos que evalúen la eficiencia a $\mathrm{P}$ en diferentes condiciones agroecológicas y etapas fenológicas del cultivo.

El objetivo de este estudio fue evaluar la variabilidad genotípica del en maíz nativo de la Meseta Puhrépecha para eficiencia y respuesta a $\mathrm{P}$ durante la etapa de floración y de esta manera probar la hipótesis de que existen variedades nativas de maíz eficientes en adquirir y utilizar el $P$ del suelo.

\section{MATERIALES Y MÉTODOS}

El presente estudio se realizó en condiciones de temporal, de mayo a diciembre de 2014. Las parcelas experimentales se ubicaron en los municipios de Charapan (19 ${ }^{\circ}$ $39^{\prime} \mathrm{N}$ y $\left.101^{\circ} 54^{\prime} \mathrm{O}\right)$, Erongarícuaro ( $19^{\circ} 35^{\prime} \mathrm{N}$ y $\left.101^{\circ} 43^{\prime} \mathrm{O}\right)$,

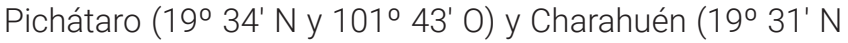
y $101^{\circ} 42^{\prime}$ O), en el estado de Michoacán. El clima es templado sub-húmedo con lluvias en verano [C (w2) (w)], con una temperatura media anual de $20{ }^{\circ} \mathrm{C}$ y altura de $2260 \mathrm{a}$ 2414 msnm (Muñoz, 2003). En el Cuadro 1 se muestran las características físico-químicas del suelo.

Los experimentos incluyeron dos niveles de fertilización con una baja (25 kg ha-1) y alta (50 kg ha-1) aplicación de
$\mathrm{P}_{2} \mathrm{O}_{5}$ proveniente de superfosfato triple, combinados con dos testigos de maíz (cv. Ocelote y un genotipo de maíz nativo de la región) y 25 genotipos de maíz provenientes del programa de mejoramiento del Instituto Nacional de Investigaciones Forestales, Agrícolas y Pecuarias (INIFAP) e integrados por genotipos nativos de la Meseta P'urhépecha, así como maíces mejorados de amplia base genética. De acuerdo con el tiempo (días) en alcanzar el $50 \%$ de floración masculina los materiales se clasificaron como: precoces (de 75 a 80 d con 33 materiales), intermedios (de 85 a 95 d con 40 materiales) y tardíos (de 95 a 110 d con 35 materiales), y en cuatro grados de mejoramiento: nativos (N), nativos mejorados (NM), híbridos $\times$ nativos $(\mathrm{H} \times \mathrm{N})$ e híbridos mejorados (S) (Cuadro 2). A todas las unidades experimentales se les aplicó una dosis de $60 \mathrm{~kg} \mathrm{~N}^{-1} \mathrm{en}$ forma de urea a la siembra y una de $60 \mathrm{~kg} \mathrm{~N} \mathrm{ha}^{-1}$ a los $42 \mathrm{~d}$ después de la siembra.

Las plantas se muestrearon en la etapa de floración. Las hojas, tallo, raíz y espiga de cada conjunto de cuatro plantas/genotipo se deshidrataron en una estufa a $65^{\circ} \mathrm{C}$ por 72 h, para luego registrar el peso seco. El tejido seco se molió en un molino Wiley-Thomas con una malla de $1 \mathrm{~mm}$ y se utilizaron sub-muestras de $70 \mathrm{mg}$ para calcinarlas en una mufla (CEM®, mod. MAS-7000, USA) a $500^{\circ} \mathrm{C}$ por $8 \mathrm{~h}$. La estimación de la concentración de P se realizó por colorimetría mediante la reacción con una solución de molibdato $\left[\left(\mathrm{NH}_{4}\right)\right.$ $\left.6 \mathrm{Mo7O}_{24}\right]$ y su medición en un espectrofotómetro de UV/VIS (Perkin Elmer ${ }^{\circledR}$, mod. Lamda 40, Uberlingen, Germany) a una longitud de $880 \mathrm{~nm}$ (Murphy y Riley, 1962). Se calculó el contenido de $\mathrm{P}\left(\mathrm{mg} \mathrm{g}^{-1}\right)$ de raíz, vástago y espiga mediante la multiplicación de la concentración de P con la materia seca acumulada en cada órgano. La eficiencia en la adquisición de fósforo (EAP) es una medida del índice de la absorción de P por unidad de peso seco de la raíz ( $\mathrm{mg} \mathrm{P} \mathrm{g}^{-1}$ ), y se calculó

Cuadro 1. Propiedades físicas y químicas de la capa superficial del suelo (de 0 a $20 \mathrm{~cm}$ ).

\begin{tabular}{lcccc}
\hline Características del suelo & Charapan & Pichátaro & Erongarícuaro & Charahuén \\
\hline Arena $(\%)$ & 63.8 & 53.7 & 49.4 & 55.1 \\
Limo $(\%)$ & 29.6 & 32.7 & 34.5 & 37.6 \\
Arcilla $(\%)$ & 7.4 & 13.6 & 15.9 & 7.3 \\
$\mathrm{pH}\left(\mathrm{H}_{2} \mathrm{O}\right) 1: 2$ & 5.2 & 6.1 & 6.5 & 5.9 \\
$\mathrm{~K}\left(\mathrm{mg} \mathrm{kg}^{-1}\right)$ & 85.5 & 64.4 & 84.8 & 436 \\
$\mathrm{Ca}\left(\mathrm{mg} \mathrm{kg}^{-1}\right)$ & 930 & 1583 & 2456 & 1684 \\
$\mathrm{Mg}\left(\mathrm{mg} \mathrm{kg}^{-1}\right)$ & 36.8 & 37.5 & 80.5 & 31.7 \\
$\mathrm{Fe}\left(\mathrm{mg} \mathrm{kg}^{-1}\right)$ & 31.2 & 57.1 & 62.8 & 39.7 \\
$\mathrm{~N}$ inorgánico $\left(\mathrm{mg} \mathrm{kg}^{-1}\right)$ & 10.6 & 18.9 & 28.9 & 12.7 \\
Materia orgánica $(\%)$ & 2.7 & 3.6 & 3.2 & 2.6 \\
CIC $\left(\mathrm{cmol} \mathrm{kg}^{-1}\right)$ & 5.3 & 14.7 & 21.1 & 12.3 \\
P disponible $\left(\mathrm{g} \mathrm{P} \mathrm{kg}^{-1}\right)$ & 0.28 & 1.22 & 6.8 & 3.2 \\
\hline
\end{tabular}


Cuadro 2. Medias del peso seco total y eficiencia en la adquisición de fósforo en variedades de maíz cultivadas en un suelo con reducida (BP) y alta (AP) dosis de fertilización fosforada. Los genotipos se ordenaron de acuerdo con el índice de eficiencia a fósforo (IEF) del peso seco total.

\begin{tabular}{|c|c|c|c|c|c|c|c|c|c|c|c|c|}
\hline \multirow[t]{2}{*}{ ID } & \multirow{2}{*}{ Variedades } & \multicolumn{6}{|c|}{ Peso seco total (g/planta) } & \multicolumn{5}{|c|}{$\begin{array}{c}\text { Eficiencia en la adquisición } \\
\text { de fósforo (mg P/g raíz) }\end{array}$} \\
\hline & & $\mathrm{M}^{+}$ & $\mathrm{GM}^{++}$ & $\mathrm{BP}$ & $\mathrm{AP}$ & IEF" & PSsd" & $\mathrm{GR}^{ \pm}$ & $\mathrm{BP}$ & $A P$ & EAPsd" & $\mathrm{GR}$ \\
\hline 75 & ZR-6 & $T$ & $N$ & 102.6 & 111.9 & 0.82 & 1.54 & $\mathrm{ER}$ & 13.3 & 16.6 & 0.72 & ER \\
\hline 117 & $\mathrm{H}-7545$ & $\mathrm{~T}$ & $\mathrm{H} \times \mathrm{N}$ & 99.9 & 92.7 & 0.52 & 0.19 & ER & 14.9 & 13.9 & -0.31 & ENR \\
\hline 63 & CB-2 & $\mathrm{T}$ & N & 100.3 & 100.8 & 0.45 & 0.68 & ER & 13.0 & 14.0 & -0.34 & ENR \\
\hline 206 & Tepetate & । & NM & 81.3 & 92.5 & 0.33 & 0.05 & $\mathrm{ER}$ & 16.8 & 15.9 & 0.37 & ER \\
\hline 6 & SHUI-2 & $\mathrm{T}$ & $N$ & 98.0 & 112.8 & 0.30 & 1.44 & ER & 13.4 & 15.0 & 0.36 & ER \\
\hline 242 & Criollo & $\mathrm{T}$ & NM & 93.8 & 98.0 & 0.24 & 0.67 & ER & 13.0 & 13.4 & -0.51 & ENR \\
\hline 214 & Mojonera & । & NM & 86.2 & 92.9 & 0.12 & 0.15 & ER & 11.9 & 12.6 & -0.73 & ENR \\
\hline 113 & Paso del Muerto & $\mathrm{T}$ & NM & 83.1 & 89.6 & 0.02 & 0.02 & ER & 14.0 & 14.1 & -0.25 & ENR \\
\hline 230 & Macho II-04 & । & $S$ & 75.1 & 98.0 & 0.01 & 0.50 & ER & 17.0 & 15.6 & 0.30 & ER \\
\hline 109 & Paso del Muerto & $\mathrm{T}$ & NM & 89.8 & 87.0 & 0.80 & -0.20 & ENR & 13.9 & 13.9 & -0.27 & ENR \\
\hline 127 & DP X Tromba & $\mathrm{T}$ & $\mathrm{H} \times \mathrm{N}$ & 93.9 & 84.8 & 0.43 & -0.31 & ENR & 14.6 & 14.6 & -0.05 & ENR \\
\hline 236 & Macho-II-03 & $\mathrm{P}$ & S & 77.7 & 84.9 & 0.18 & -0.33 & ENR & 15.7 & 16.4 & 0.59 & ER \\
\hline 62 & $\mathrm{PICH}-1$ & । & N & 78.4 & 82.5 & 0.15 & -0.41 & ENR & 16.6 & 13.6 & -0.39 & ENR \\
\hline 17 & ZPT-2 & $\mathrm{P}$ & $N$ & 79.4 & 83.8 & 0.05 & -0.44 & ENR & 16.1 & 18.2 & 1.04 & ER \\
\hline 115 & Santa Clara & $\mathrm{T}$ & NM & 87.6 & 100.9 & -0.10 & 0.70 & NER & 13.7 & 13.5 & -0.43 & NENR \\
\hline 199 & CCHEDE & $P$ & NM & 74.6 & 93.0 & -0.16 & 0.03 & NER & 13.5 & 15.3 & 0.23 & NER \\
\hline 124 & Corupo & $\mathrm{T}$ & NM & 80.0 & 94.3 & -0.48 & 0.28 & NER & 13.4 & 13.3 & -0.52 & NENR \\
\hline 241 & Macho-IV-05 & $\mathrm{T}$ & S & 79.6 & 93.4 & -0.50 & 0.14 & NER & 12.6 & 15.2 & 0.19 & NER \\
\hline 243 & Ocelote & । & $\mathrm{CV}$ & 82.3 & 92.8 & -0.52 & 0.04 & NER & 15.0 & 11.3 & -1.21 & NENR \\
\hline 181 & Macho-III-04 & । & S & 70.3 & 76.2 & -0.06 & -0.90 & NENR & 17.0 & 15.6 & 0.35 & NER \\
\hline 193 & $\mathrm{NACl}-1$ & $P$ & $\mathrm{H} \times \mathrm{N}$ & 80.8 & 81.3 & -0.20 & -0.54 & NENR & 17.0 & 17.3 & 0.90 & NER \\
\hline 185 & CIMMYT-1 & $P$ & $\mathrm{H} \times \mathrm{N}$ & 73.0 & 72.0 & -0.21 & -1.21 & NENR & 15.2 & 16.1 & 0.37 & NER \\
\hline 140 & Cruz Gorda & $P$ & NM & 75.4 & 87.8 & -0.24 & -0.19 & NENR & 14.5 & 16.1 & 0.42 & NER \\
\hline 135 & HV-313XDE & $\mathrm{P}$ & $\mathrm{H} \times \mathrm{N}$ & 65.7 & 78.3 & -0.27 & -0.69 & NENR & 15.1 & 14.5 & -0.11 & NENR \\
\hline 189 & El Tigre & $P$ & NM & 74.9 & 78.0 & -0.30 & -0.87 & NENR & 15.4 & 15.4 & 0.15 & NER \\
\hline 99 & TZU-7 & $\mathrm{P}$ & $N$ & 73.2 & 84.1 & -0.68 & -0.24 & NENR & 14.2 & 12.2 & -0.88 & NENR \\
\hline 234 & Macho-I-04 & $\mathrm{P}$ & $S$ & 72.2 & 87.9 & -0.71 & -0.09 & NENR & 13.7 & 14.9 & 0.02 & NER \\
\hline
\end{tabular}

${ }^{\dagger}$ M: Madurez: Precoz (P), Intermedio (I) y Tardío (T). ${ }^{+\dagger}$ GM: Grupo de Mejoramiento: Nativo (N), Nativo Mejorado (CM), Híbrido $\times$ Nativo (H×N), Sintético (S), Cultivar (cv). "IEF: Índice de eficiencia a fósforo, obtenido del análisis de componentes principales. "PSsd, EAPsd: Valor estandarizado del peso seco total y eficiencia en la adquisición de P en condiciones de AP. GR: Respuesta a fósforo; se expresó como el peso seco total o EAP en condiciones de AP. Se definieron cuatro categorías: eficiente y responde (ER), eficiente y no responde (ENR), no eficiente y responde (NER), no eficiente y no responde (NENR)

con base en la relación del contenido total de P y el peso seco de la raíz en gramos (Fageria et al., 2008).

Se empleó un diseño de bloques completos al azar con un arreglo de tratamientos en parcelas divididas con cinco repeticiones por tratamiento. Los niveles de $\mathrm{P}$ correspondieron a la parcela principal y los genotipos, distribuidos aleatoriamente dentro de cada parcela, correspondieron a las sub-parcelas. Cada unidad experimental consistió de dos surcos de $5 \mathrm{~m}$ de longitud por genotipo. Las semillas se sembraron a una profundidad de $6 \mathrm{~cm}$ y a una distancia de $25 \mathrm{~cm}$ entre cada planta y a $60 \mathrm{~cm}$ de distancia entre cada surco. Las diferencias entre genotipos en respuesta a los niveles de $\mathrm{P}$ aplicados se evaluaron mediante análisis de varianza (SAS, 2000). Previo al análisis se realizó una prueba de Bartlett para la homogeneidad de varianza en todas las variables incluidas (SAS, 2000). La varianza del error fue heterogénea para algunas variables, así que los datos fueron transformados a base logarítmica y raíz cuadrada (SAS, 2000). Se utilizó la prueba de Tukey con valores 
de $P \leq 0.05$ para comparar las diferencias entre genotipos y tratamientos de $\mathrm{P}$ en las distintas variables evaluadas.

La eficiencia a P, definida como el crecimiento sostenido en condiciones de baja disponibilidad de $P$, se determinó de acuerdo con el índice de eficiencia a P (IEF) (Pan et al., 2008) obtenido mediante un análisis de componentes principales (ACP) a partir de los datos estandarizados de crecimiento en condiciones de bajo contenido de $P(B P)$ y relativo a la condición en alto contenido de $P(A P)$ (SAS, 2000). Los valores del IEF de cada genotipo se calcularon de acuerdo con el componente principal retenido $(C P)$ y su contribución relativa $(C R): \mathrm{IEF}={ }^{27} \sum_{i=1} C P_{i} \times C R_{i}$.

Posteriormente, con los datos de IEF por genotipo se hizo un análisis de conglomerados para agrupar por categorías de eficiencia [eficientes (E), moderadamente eficientes (ME) e ineficientes (I)], y en cuatro categorías de respuesta a P de acuerdo con el IEF en combinación con la respuesta a P (peso seco total y EAP en AP) [eficientes y con respuesta (ER); no eficientes y con respuesta (NER), no eficientes y sin respuesta (NENR), y eficientes y sin respuesta (ENR)]. El análisis de conglomerados se realizó según el método de Ward (Romerburg, 1984).

\section{RESULTADOS Y DISCUSIÓN}

Según el análisis de componentes principales (CP), de las características de crecimiento de los genotipos evaluados los componentes CP1 al CP4 contribuyeron con 30, 20, 18 y $12 \%$ de la variación entre grupos. Estos componentes en conjunto aportaron $80 \%$ de la variación total observada. La variabilidad del primer componente (CP1) estuvo determinada por valores positivos de: peso seco de la raíz (0.87), peso seco de vástago (0.87) y peso seco total (0.90) en condiciones de bajo fósforo (BP). El componente 2 (CP2) estuvo influenciado positivamente por el contenido de $\mathrm{P}$ en vástago (0.67) en BP y los valores relativos a la condición de alto contenido de P (AP) en vástago (0.81) y espiga (0.67). En el componente 3 (CP3) ejercieron mayor peso los índices relativos del peso seco de vástago (0.74), peso seco total (0.69) y eficiencia en adquisición de P (EAP) (0.62). En el componente 4 (CP4) influyó positivamente el peso seco de la espiga (0.86).

En la Figura 1 se presenta la formación de los grupos de eficiencia (combinada de las cuatro localidades) con base en sus parámetros de crecimiento en BP y sus valores relativos al tratamiento de AP. El Grupo 1 (Índice de eficiencia a $P$, IEF < 0.60) consistió de cinco genotipos eficientes, de los cuales 20 y $80 \%$ fueron de madurez intermedia y tardía. El Grupo $2(0.11<$ IEF <-0.39) estuvo compuesto por 11 genotipos moderadamente eficientes con 27, 19 y $54 \%$ de madurez precoz, intermedia y tardía. El Grupo 3 (IEF <-0.39) lo integraron 11 genotipos ineficientes a P, la mayoría de los cuales fueron variedades precoces (64\%). La comparación de la eficiencia a $\mathrm{P}$ combinada de los 27 genotipos mostró que la clasificación de los genotipos evaluados fue constante en los tres grupos de eficiencia. Los genotipos 63, 75, 109, 117 y 127 fueron los más eficientes a P. Estos genotipos acumularon mayor peso y contenido de $\mathrm{P}$ en la condición de BP (Figuras 2 y 3).

Los 27 genotipos se separaron en cuatro grupos de acuerdo con su respuesta a P (expresada por el peso seco total y EAP en AP, estandarizado) e IEF. Las categorías fueron: (i) eficientes y con respuesta (ER), (ii) ineficientes y con respuesta (NER), (iii) ineficientes y sin respuesta (NENR) y (iv) eficientes y sin respuesta (ENR) (Figura 4). En la comparación de la respuesta y eficiencia a $\mathrm{P}$ se identificaron nueve genotipos eficientes en $\mathrm{BP}$, que respondieron a la mayor aplicación del mismo (ER) (Figura 4; Cuadro 2). Los genotipos $6,63,75,113,117,206,214,230$ y 242 (testigo maíz nativo) exhibieron una similar acumulación del peso seco total en ambos ambientes.

Cinco genotipos (115, 124, 199, 241 y 243) (testigo cv. Ocelote) presentaron una alta respuesta a $\mathrm{P}$, manifestada por un incremento en el peso seco total pero que fueron ineficientes en la acumulación de materia seca en BP; mientras que ocho genotipos $(99,135,140,181,185,189$, 193 y 234) fueron ineficientes a BP y no respondieron a la condición de AP. En la categoría eficientes y sin respuesta (ENR) destacan cinco genotipos $(17,62,109,127,236)$ de moderada y alta eficiencia, que incrementaron el peso seco total en el ambiente de BP, pero no respondieron a la mayor disponibilidad del mismo. En la clasificación combinada de las cuatro localidades se determinó que los genotipos SHUI-2 (6), ZR-6 (75), Paso del Muerto (113), Tepetate (206) y Macho II-04 (230) fueron los más eficientes en BP y que también respondieron a la alta fertilización.

En este estudio se adoptaron dos índices para seleccionar genotipos eficientes a P. El primero estimó el crecimiento relativo del cultivo obtenido en condiciones limitantes de $P$, con respecto al crecimiento alcanzado en condiciones nutrimentales óptimas (Fageria et al., 2008). Adicionalmente, se utilizó el sistema propuesto por Gerloff (1977) para clasificar los genotipos de maíz con base en el crecimiento o acumulación de $\mathrm{P}$ en condiciones óptimas y de deficiencia. Este sistema incluyó genotipos: 1) Eficientes y con respuesta (ER), plantas con mayor crecimiento con reducida disponibilidad de $\mathrm{P}$ y que respondieron a los altos niveles de fertilización; 2) Ineficientes y con respuesta (NER), plantas con reducido crecimiento en $\mathrm{BP}$ y con alta respuesta a la adición del nutriente; 3) Eficientes y sin respuesta (ENR), plantas con alto crecimiento en bajo nivel de $\mathrm{P}$, pero que no responden a la adición de $P ;$ y 4) Ineficientes y sin respuesta (NENR), 
plantas con reducido crecimiento en BP y sin respuesta a condiciones de alto nivel nutrimental.

Con este sistema de categorización fue posible obtener información para comparar los genotipos que se desarrollaron en condiciones de deficiencia de P en el suelo (eficientes vs. ineficientes) y condiciones adecuadas de fertilización (con respuesta vs. sin respuesta) (Figura 4). Los resultados obtenidos sugieren que el uso de ambos sistemas es útil para identificar germoplasma eficiente a $\mathrm{P}$ y contrastar su eficiencia en diferentes condiciones edáficas (Bayuelo-Jiménez y Ochoa-Cadavid, 2014; Manschadi et al., 2014)

En la comparación de la eficiencia en la adquisición de fósforo (EAP), hubo una reducida variación en la distribución de

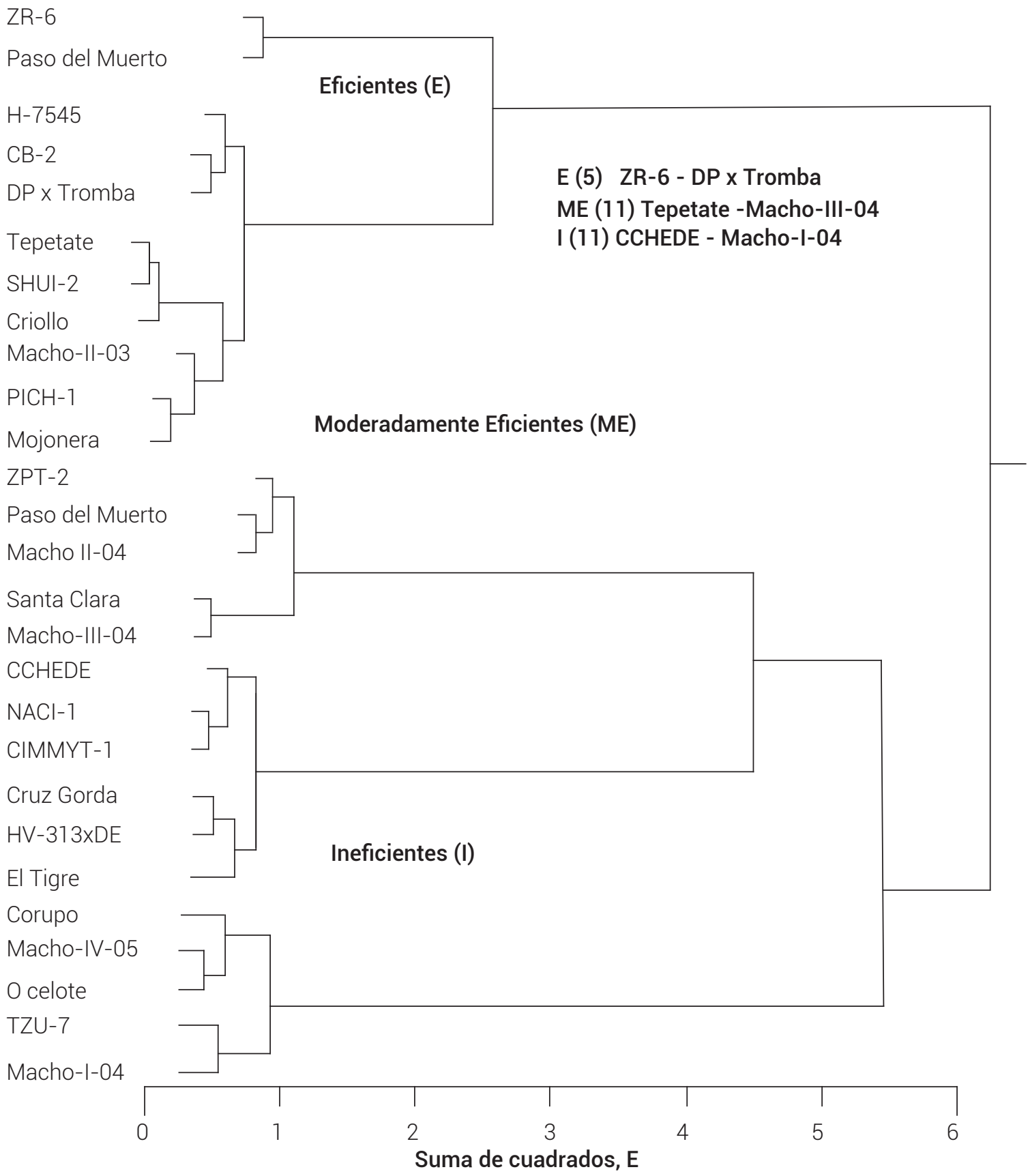

Figura 1. Clasificación combinada de 27 genotipos de maíz de acuerdo con el índice de eficiencia a fósforo (IEF), mediante el método de agrupación de Ward. Los grupos I, ME y E representan los genotipos ineficientes (11), moderadamente eficientes (11) y eficientes (5). 

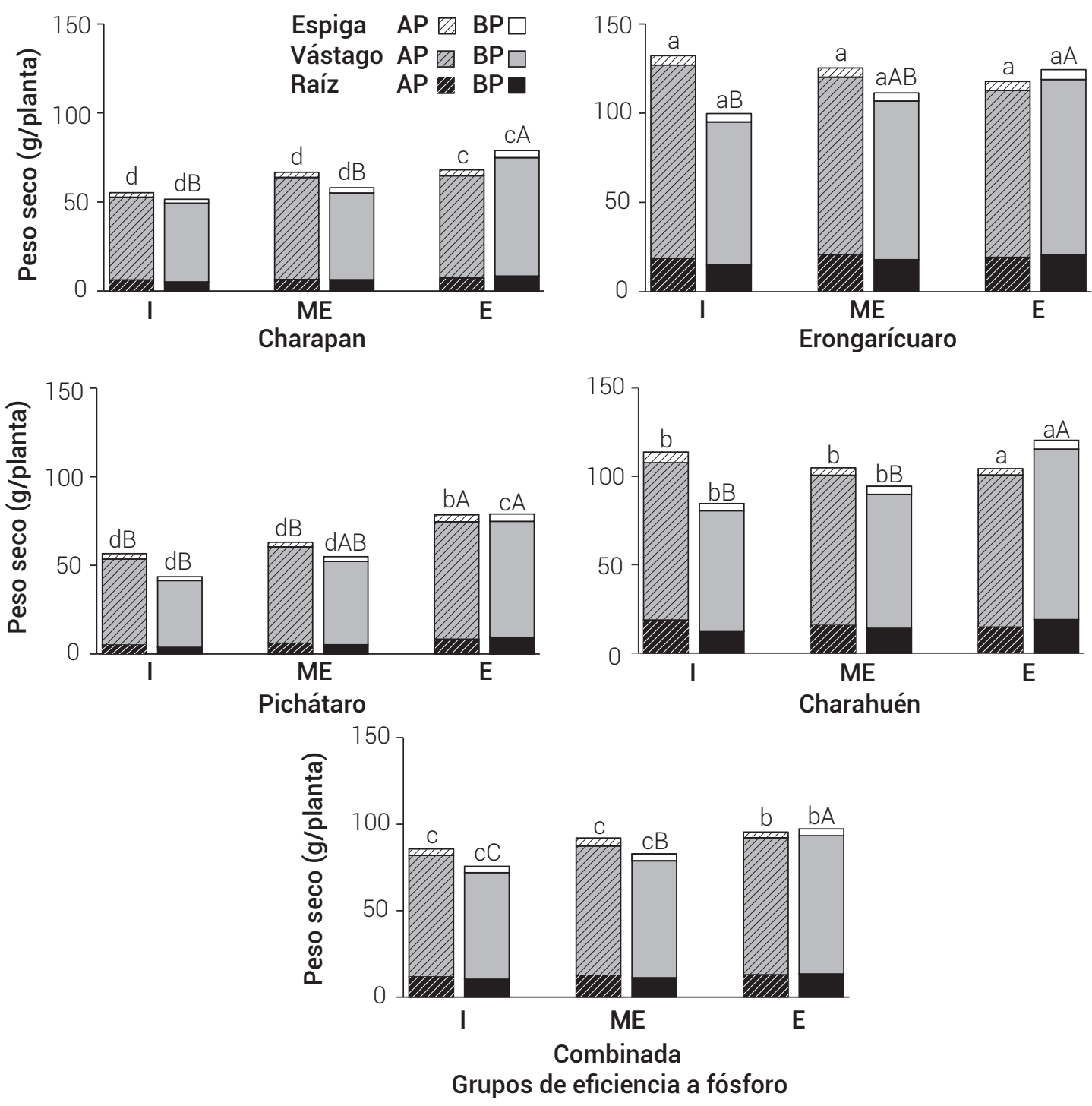

Figura 2. Peso seco de la raíz, vástago y espiga en condiciones de reducida (I), moderada (ME) y alta eficiencia (E) a fósforo. Las barras representan la media de 11, 11 y 5 genotipos. Las medias con letras iguales entre localidades (minúsculas) y entre grupos de eficiencia (mayúsculas) por nivel de fósforo son estadísticamente iguales entre sí (Tukey, 0.05).

los genotipos. Mientras que los genotipos 6, 75, 206, 230 y 236 se mantuvieron como los más eficientes en la EAP en ambos ambientes; los genotipos 63, 113, 117, 214 y 242 (testigo, maíz nativo de la región) se clasificaron como los más eficientes en BP, pero con una reducida respuesta a la condición de AP. Estos genotipos fueron muy eficientes para adquirir este nutriente en $\mathrm{BP}$, pero no respondieron a la alta adición del mismo. La comparación de la eficiencia y respuesta a P basada en el peso seco total y EAP identificó a cuatro genotipos eficientes en BP y que respondieran a la alta dosis de fertilización. Los resultados de este estudio mostraron que existe variación genotípica en maíz nativo para eficiencia en la adquisición de P, y que esta variación está acentuada en los genotipos de maduración tardía.
La eficiencia en el uso de fósforo (EUP) es el producto de la eficiencia en la adquisición (EAP; P total en la planta por unidad de nutriente suministrado) y utilización de P (EUtP; el crecimiento y rendimiento del cultivo por unidad de $\mathrm{P}$ absorbido) (Wang et al., 2010). Una mayor EUP puede conseguirse mediante estrategias que mejoren la exploración, adquisición y removilización de $\mathrm{P}$ del suelo (Manschadi et al., 2014; Simpson et al., 2011). Debido a la relativa inmovilidad y heterogénea distribución de $\mathrm{P}$ en la capa arable del suelo, la configuración de las raíces en espacio y tiempo son características clave para optimizar su adquisición (Lynch, 2013).

El sistema radical de maíz está conformado por raíces 


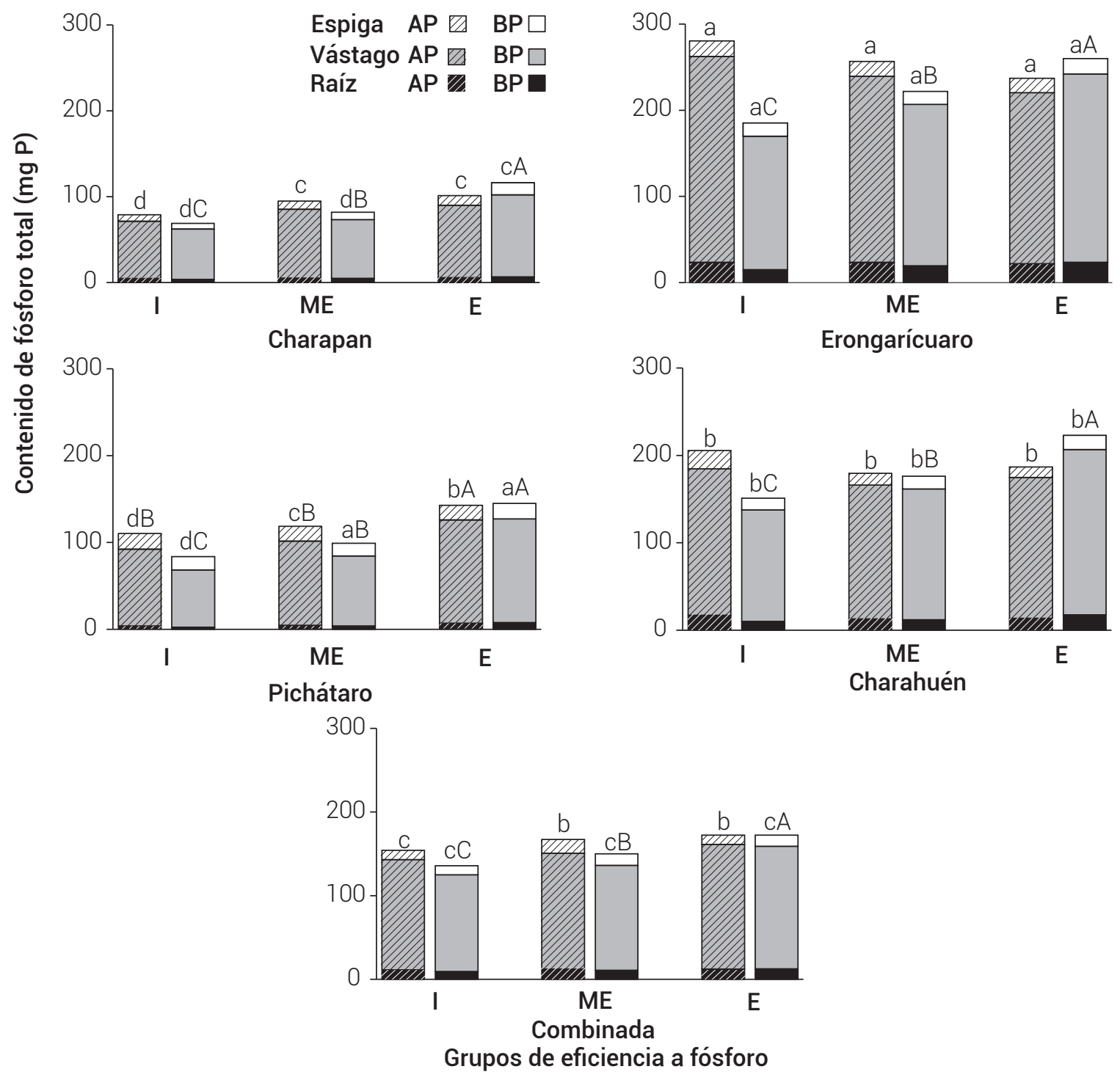

Figura 3. Contenido de fósforo en raíz, vástago y espiga en condiciones de reducida (I), moderada (ME) y alta eficiencia (E) a fósforo. Las barras representan las medias de 11, 11 y 5 genotipos. Las medias con letras iguales entre localidades (minúsculas) y entre grupos de eficiencia (mayúsculas) por nivel de fósforo son estadísticamente iguales entre sí (Tukey, 0.05).

nodales que absorben los nutrientes móviles e inmóviles del suelo y que responden diferencialmente al estrés impuesto por el ambiente (Postma et al., 2014). Los genotipos eficientes que incrementan el número de raíces nodales en condiciones de reducida disponibilidad de $\mathrm{P}$ tienen una mayor capacidad para mantener un crecimiento continuo y para adquirir $P$ en la superficie del suelo (Zhu et al., 2005). El ángulo de las raíces axiales, el número de raíces adventicias, el número de raíces seminales y nodales, la densidad y longitud de pelos radicales y la formación de aerénquima, son algunas de las características que favorecen la adquisición de P del suelo (Simpson et al., 2011). En maíces nativos de la Meseta P'urhépecha, la mayor absorción de P del suelo está mediada por el incremento del número de raíces nodales, una mayor ramificación de raíces laterales y el desarrollo de pelos radicales más densos y largos (Bayuelo-Jiménez et al., 2011).

La asociación con hongos micorrícicos arbusculares (HMA) también incrementa la superficie de absorción de la raíz y, por tanto, la adquisición de nutrientes minerales como P, Cu y Zn. Sin embargo, los HMA son ubicuos y están menos sujetos a selección. A diferencia de las características que definen la arquitectura de la raíz, la información sobre la variabilidad genotípica de HMA es reducida. Además, los resultados de diversas investigaciones sugieren que las características de la raíz actúan de forma aditiva y sinérgica con los HMA (Lynch, 2013). 

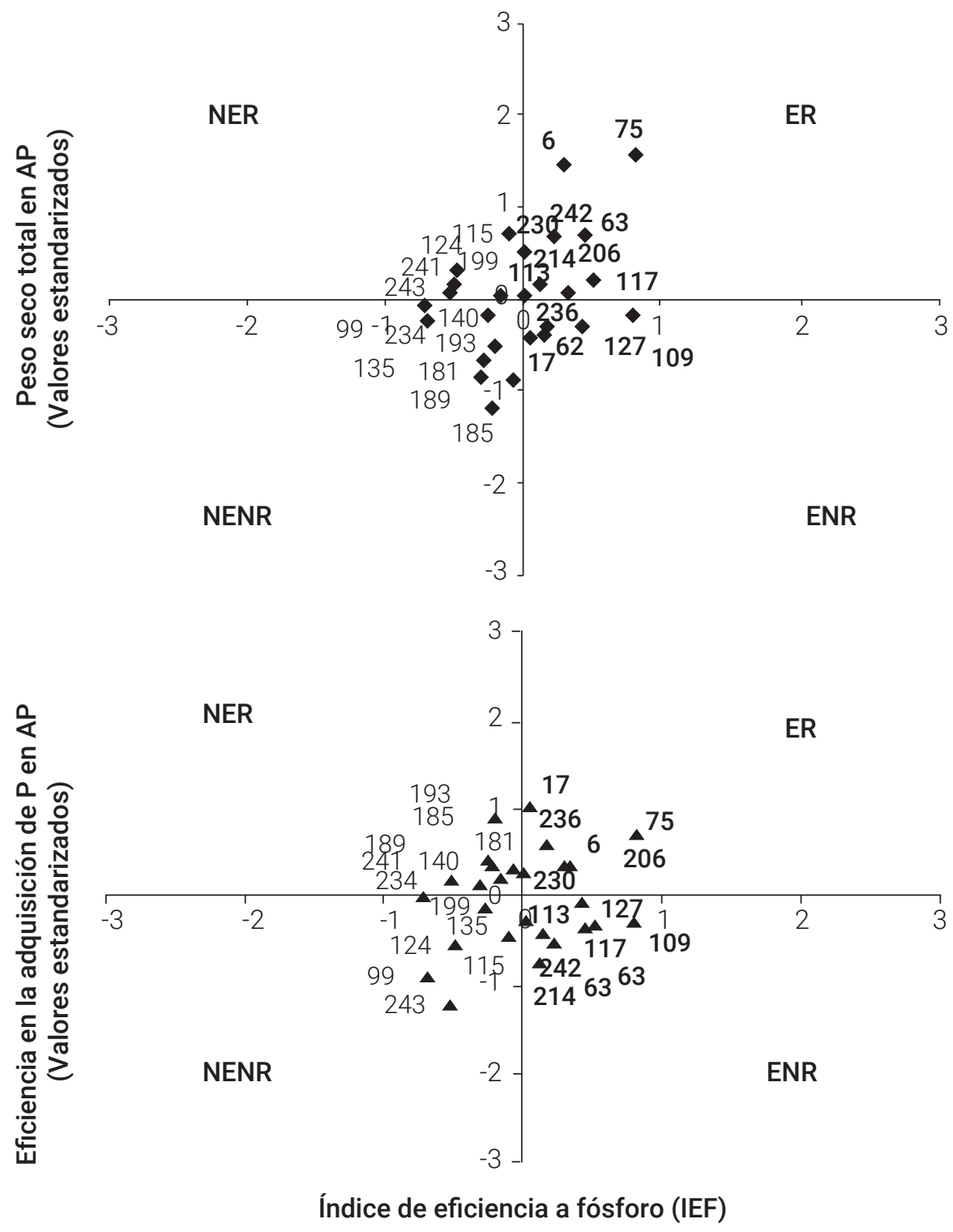

Figura 4. División de los 27 genotipos de maíz de acuerdo con el índice de eficiencia a fósforo (IEF) y de los valores estandarizados del peso seco total y la eficiencia en la adquisición de fósforo (EAP) en AP. Los valores estandarizados del peso seco total y EAP se estimaron de acuerdo con la siguiente función: $X s=(X-X) / S D$. Las categorías están representadas con genotipos eficientes con respuesta (ER), no eficientes con respuesta (NER), eficientes sin repuesta (ENR) y no eficientes y sin respuesta (NENR), respectivamente. Se indica el número de cada genotipo.

La eficiencia en la adquisición de fósforo (EAP) está fuertemente afectada por el estado hídrico del suelo (McBeath et al., 2012). Los prolongados periodos de sequía combinados con altas temperaturas afectan la productividad agrícola al reducir la eficiencia en la adquisición de nutrientes (Lynch, 2013). El déficit hídrico, reduce el crecimiento de la raíz y la EAP al alterar el transporte de $\mathrm{P}$ en el suelo. En condiciones de déficit hídrico, la eficiencia del fertilizante fosforado puede disminuir hasta $3 \%$, mientras que durante el periodo de lluvia incrementa hasta $30 \%$ (McBeath et al., 2012). A pesar de que no se evaluó el efecto del estrés hídrico, es plausible que éste tenga implicaciones en la productividad de los sistemas agrícolas de temporal de ésta y otras regiones de México. El desarrollo de genotipos eficientes en el uso de P y agua son aspectos importantes en la sostenibilidad económica y ambiental de este sector agrícola. 
Aunado a la configuración y morfología de la raíz, la fenología de la planta beneficia la absorción de nutrientes del suelo (Lynch, 2013). La fenología determina la duración del periodo de adquisición y utilización de los nutrientes y sincroniza las relaciones de fuente y demanda del recurso (Nord y Lynch, 2009). Una mayor duración del crecimiento del vástago promueve una mayor utilización fisiológica del nutriente (Bayuelo-Jiménez y Ochoa, 2014). Los resultados confirman que independientemente de la localidad estudiada, los genotipos eficientes de maduración tardía acumularon mayor peso y contenido de $\mathrm{P}$ en el vástago y espiga que los genotipos ineficientes, mayormente precoces (Figuras 2 y 3). En la mayoría de las condiciones de cultivo, la disponibilidad de $\mathrm{P}$ en la solución del suelo fue limitante $\left(0.28\right.$ a $\left.6.8 \mathrm{mg} \mathrm{P} \mathrm{kg}^{-1}\right)$, pero los genotipos tardíos tuvieron una mayor oportunidad de adquirir $\mathrm{P}$ durante más tiempo.

\section{CONCLUSIONES}

Los genotipos de maíz de la Meseta P'urhépecha mostraron una gran variabilidad en el crecimiento vegetal, eficiencia y respuesta a fósforo, en etapa de floración. La eficiencia a fósforo estuvo determinada por una mayor producción de materia seca en la raíz, el vástago y la espiga; una mayor asignación de fósforo hacia los vástagos, y una mayor eficiencia en la absorción de fósforo por unidad de peso seco de la raíz. La maduración tardía de los genotipos eficientes favoreció una mayor adquisición de fósforo y crecimiento en la planta. Estos resultados indican que existe variabilidad genotípica para la eficiencia a fósforo en el germoplasma de maíz.

\section{AGRADECIMIENTOS}

Al Consejo Nacional de Ciencia y Tecnología, FOMIXGobierno del Estado de Michoacán MICH-2012 No. 197142/2013-2015 y la Coordinación de Investigación Científica, UMSNH 6.11/2015-2016.

\section{BIBLIOGRAFÍA}

Alcalá J. M., C. A. Ortiz-Solorio y M. C. Gutiérrez-Castorena (2001) Clasificación de los suelos de la Meseta Tarasca, Michoacán. Terra 19:217-239.

Bayuelo-Jiménez J. S. and I. Ochoa-Cadavid (2014) Phosphorus acquisition and internal utilization efficiency among maize landraces from the central Mexican highlands. Field Crops Research 156:123-134.
Bayuelo-Jiménez J. S., V. A. Pérez-Decelis, M. L. Magdaleno-Armas, M. Ochoa y R. Cárdenas-Navarro (2012) Eficiencia a fósforo del germoplasma de maíz de la meseta P'urhépecha en etapa de plántula. Revista Fitotecnia Mexicana 35:199-208.

Bayuelo-Jiménez J. S., V. A. Pérez-Decelis, M. L. Magdaleno-Armas, M. Gallardo-Valdéz, I. Ochoa and J. P. Lynch (2011) Genotypic variation for root traits of maize (Zea mays L.) from P'urhépecha Plateau, under contrasting phosphorus availability. Field Crops Research 121:350-362.

Fageria N. K., V. C. Baligar and Y. C. Li (2008) The role of nutrient efficient plants in improving crop yields in the twenty first century. Journal of Plant Nutrition 1:1121-1157.

Gerloff G. C. (1977) Plant efficiencies in the use of N, P and K. In: Plant Adaptation to Mineral Stress in Problem Soils. M. J. Wright (ed.) Cornell University Press New York. pp:161-174.

Lynch J. P. (2013) Steep, cheap and deep: an ideotype to optimize water and $\mathrm{N}$ acquisition by maize root systems. Annals of Botany 112:347-357

Manschadi A. M., K. Hans-Peter, J. Vollmann, J. Eitzinger and W. Wenzel (2014) Developing phosphorus-efficient crops varieties-An interdisciplinary research framework. Field Crops Research 162:8798.

McBeath T. M., M. J. McLaughlin, J. K. Kirby and R. M. Armstrong (2012) The effect of soil water status on fertilizer, topsoil and subsoil phosphorus utilization by wheat. Plant and Soil 358:337-348.

Muñoz 0. A. (2003) Descifrando la diversidad del maíz en los nichos ecológicos de México. En: Centli Maíz (Prehistoria e historia diversidad, potencial, origen genético y geográfico). Colegio de Postgraduados, Montecillo, México. pp:83-159.

Murphy J. and J. Riley (1962) A modified single solution method for the determination of phosphate in natural waters. Analytical Chemical Acta 27:31-36.

Newton A. C., T. Akar, P. Baresel, P. J. Bebeli, E. Bettencourt, K. V. Bladenopoulos, J. H. Czembor, D. A. Fasoula, A. Katsiotis, K. Koutis, G. Kovacs, H. Larsson, M. A. Pinheiro de Carvalho, D. Rubiales, J. Russel, T. M. Dos Santos and M. C. Vaz Patto (2010) Cereal landraces for sustainable agriculture: A review. Agronomy Sustainable Development 30:237-269.

Nord E. A. and J. P. Lynch (2009) Plant phenology: a critical controller of soil resource acquisition. Journal of Experimental Botany 60:1927-1937.

Pan X. W., W. B. Li, Q. Y. Zhang, Y. H. Li and M. S. Liu (2008) Assessment on phosphorus efficiency characteristics of soybean genotypes in phosphorus deficient soils. Agricultural Science in China 7:958969

Postma J. A., A. Dathe and J. P. Lynch (2014) The optimal lateral root branching density for maize depends on nitrogen and phosphorus availability. Journal of Plant Physiology 166:590-602.

Romerburg H. C. (1984) Cluster Analysis for Researches. Lifetime Learning Publications Belmont, California. $277 \mathrm{p}$.

SAS (2000) SAS User's Guide: Statistics. SAS Institute, Inc., Cary, NC.

Simpson R. J., A. Oberson, R. A. Culvenor, M. H. Ryan, E. J. Veneklaas, H. Lambers, J. P. Lynch, P. R. Ryan, E. Delhaize, F. A. Smith, S. E. Smith, P. R. Harvey and A. E. Richardson (2011) Strategies and agronomic interventions to improve the phosphorus-use efficiency of farming systems. Plant and Soil 349:89-120.

Wang X., J. Shen and H. Liao (2010) Plant acquisition or utilization, which is more critical for enhancing phosphorus efficiency in modern crops. Plant Science 179:302-306

Zhu J., S. M. Kaeppler and J. P. Lynch (2005) Topsoil foraging and phosphorus acquisition efficiency in maize (Zea mays L.). Functional Plant Biology 32:1-14. 
